\title{
Modified Function Projective Synchronization of Fractional Order Chaotic Systems with Different Dimensions
}

\author{
Hong-Juan Liu, ${ }^{1,2}$ Zhi-Liang Zhu, ${ }^{1}$ Hai Yu, ${ }^{1}$ and Qian Zhu ${ }^{1,2}$ \\ ${ }^{1}$ Software College, Northeastern University, Shenyang 110819, China \\ ${ }^{2}$ School of Information Science \& Engineering, Northeastern University, Shenyang 110819, China \\ Correspondence should be addressed to Hong-Juan Liu; liuhongjuan0125@163.com
}

Received 23 February 2013; Revised 23 September 2013; Accepted 27 September 2013

Academic Editor: Daniele Fournier-Prunaret

Copyright (C) 2013 Hong-Juan Liu et al. This is an open access article distributed under the Creative Commons Attribution License, which permits unrestricted use, distribution, and reproduction in any medium, provided the original work is properly cited.

\begin{abstract}
The modified function projective synchronization of different dimensional fractional-order chaotic systems with known or unknown parameters is investigated in this paper. Based on the stability theorem of linear fractional-order systems, the adaptive controllers with corresponding parameter update laws for achieving the synchronization are given. The fractional-order chaotic system and hyperchaotic system are applied to achieve synchronization in both reduced order and increased order. The corresponding numerical results coincide with theoretical analysis.
\end{abstract}

\section{Introduction}

Synchronization has attracted a great deal of interest due to its important applications in ecological systems [1], physical systems [2], chemical systems [3], modeling brain activity, system identification, pattern recognition phenomena and secure communications [4], and so forth. Since the pioneering work of Pecora and Carroll [5], various synchronization scenarios have been studied for chaotic systems, including complete synchronization [6], phase synchronization [7], lag synchronization [8], Q-S synchronization [9], and projective synchronization [10]. As a much more universal synchronization manner, the modified function projective synchronization (MFPS) means that the drive and response systems could be synchronized up to a scaling function matrix, but not a constant matrix. Obviously, the unpredictability of the scaling functions in MFPS can additionally enhance the security of communication [11-13].

In recent years, the study on the nonlinear dynamics and synchronization control of fractional-order chaotic systems has become a hot topic in nonlinear research area. It is demonstrated that many fractional-order differential systems behave chaotically or hyperchaotically, such as the fractionalorder Chua circuit [14], the fractional-order Arneodo system [15], the fractional-order Chen system [16], the fractionalorder hyperchaotic Lorenz system [17], the fractional-order hyperchaotic Lü system [18], and so forth. Studies show that a fractional-order controller can provide better performances than an integer order one and lead to more robust control performance [19]. According to different definitions of the fractional-order differential equation from the integer order differential equation, most of the methods and results of chaos synchronization in the ordinary differential systems cannot be simply extended to the case of the fractional-order systems. Some approaches have been proposed to achieve chaos synchronization in fractional-order chaotic systems, such as active control [20], adaptive control [21], a scalar transmitted signal method [22], sliding mode control [23], track control [24] and fuzzy logic constant control [25], and so forth. From above and the other related literatures, one can see that, like the studies on the synchronization of integerorder chaotic systems, the synchronization in fractionalorder chaotic systems is still the dominant one among the various research of fractional-order systems.

At present, many existing schemes focus on synchronization of the fractional-order systems with the same dimension. However, in many real systems, chaos synchronization between different dimensional systems usually occurs, especially in biological and social sciences [26, 27]. Therefore, the synchronization of different dimensional fractional-order systems becomes a meaningful problem. To the best of 
our knowledge, only a few conclusions focus on the chaos synchronization of fractional-order systems with different dimensions. In [28, 29], Wang et al. advised two methods for hybrid projective synchronization of fractional-order chaotic systems with different dimensions. Hybrid projective synchronization is a different projective synchronization mode, in which the scaling factor matrix is not a diagonal matrix and a state variable in drive system can synchronize to multiple variables in response system. The synchronization mode varies from traditional synchronization concept and is devoid of generality in real applications.

In this paper, we discussed the MFPS problem between different dimensional fractional-order chaotic systems with known or unknown parameters. By reduced order or added order, the problem is transformed to the MFPS between identical dimension chaotic systems. Based on adaptive control method and stability theory of fractional-order systems, an effective controller and a parameter update law for MFPS are proposed by rigorous theoretical analysis. Two groups of examples are considered and their numerical simulations are performed. Numerical simulations verify that MFPS really can occur between different dimension fractional systems.

\section{A MFPS Scheme in Fractional-Order Chaotic System with Different Dimensions}

2.1. The Reduced-Order and Added-Order Schemes. There are several definitions of fractional derivatives. The Caputo derivative is more popular in the real applications, because the inhomogeneous initial conditions are allowed if such conditions are necessary. The Caputo fractional derivative is defined as

$$
\frac{d^{q} f(t)}{d t^{q}} \equiv D^{q} f(t)=\frac{1}{\Gamma(m-q)} \int_{0}^{t}(t-\tau)^{m-q-1} f^{(m)}(\tau) d \tau,
$$

where $m$ is the smallest integer larger than $q, D^{q}$ denotes the Caputo definition of the fractional derivative, $f^{(m)}(t)$ is the $m$-order derivative in the usual sense, and $\Gamma$ stands for gamma function.

Now, consider the fractional-order drive and response systems are described as system (2) and (3), respectively:

$$
\begin{aligned}
D^{q_{d}} X_{1} & =f_{1}\left(X_{1}\right), \\
D^{q_{r}} Y & =g(Y, \theta),
\end{aligned}
$$

where $X_{1} \in R^{m}, Y \in R^{n}, q_{d} \in R^{m}$, and $q_{r} \in R^{n}$ are fractionalorder satisfying $0<q_{d}<1,0<q_{r}<1$ and $f_{1}: R^{m} \rightarrow$ $R^{m}$ and $g: R^{n} \rightarrow R^{n}$ are two different continuous vector functions. Vector $\theta$ is system parameters of response system.

When $m=n$ and $f_{1}=g$, the drive system is identical to the response system, and this kind of synchronization problem has been well studied. However, when the order of the response system is not equal to that of the drive system, reduced order for the case $m>n$ and added order for the opposite case $m<n$ can be introduced to solve the dimensional diversity.
Case $I$ (the reduced-order scheme when $m>n$ ). When the orders of two systems satisfy the condition $m>n$ (of course $f_{1} \neq g$ ), that is, the order of the drive system is greater than that of the response system, the synchronization can be attained by reducing the order of drive system, in which the controlled response system synchronize to the projection of the drive system. Therefore, we can divide the drive system into two parts. One is the projection:

$$
D^{q_{r}} X=f\left(X_{1}\right)
$$

where $X \in R^{n}$ and $f: R^{m} \rightarrow R^{n}$. The rest is

$$
D^{q_{s}} X_{s}=f_{s}\left(X_{1}\right),
$$

where $X_{s} \in R^{s}, q_{s} \in R^{s}$, and $f_{s}: R^{m} \rightarrow R^{s}$ and orders $n, s$ satisfy $n+s=m$.

In order to unify the order of drive and response system, we can choose arbitrary $n$ state variables of drive system to project. Hereby, the possible number of projection (4) is $C_{m}^{n}=$ $m ! /(n !(m-n) !)$.

Case II (the added-order scheme when $m<n$ ). When the order of the drive system is lower than that of the response system, we can increase the order of drive system and construct an auxiliary state vector with order $n-m$ which is the function of state vector of drive system. Obviously, the defined function may also be constant function. Therefore, we define $X_{2}=\left(x_{m+1}, \ldots, x_{n}\right)$ and $f_{2}\left(X_{1}\right)=$ $\left(\phi_{1}\left(X_{1}\right), \phi_{2}\left(X_{1}\right), \ldots, \phi_{n-m}\left(X_{1}\right)\right)$, and then we get a new $n$ dimension state vector $X=\left(x_{1}, \ldots, x_{m}, x_{m+1}, \ldots, x_{n}\right)$. The drive system is rewritten as follows:

$$
D^{q_{r}} X=f\left(X_{1}\right)
$$

where $X=\left(\begin{array}{l}X_{1} \\ X_{2}\end{array}\right), f\left(X_{1}\right)=\left(\begin{array}{l}f_{1}\left(X_{1}\right) \\ f_{2}\left(X_{1}\right)\end{array}\right), X_{2} \in R^{n-m}, f_{2}: R^{m} \rightarrow$ $R^{n-m}$.

After the above transformation, the dimension of drive system (4) or (6) comes the same as response system (3) via reduced order or added order. Thus, the aim of MFPS is to design a suitable controller, which is able to synchronize the state of the transformed drive system and the response system up to different arbitrary scaling functions.

2.2. A MFPS Strategy of Fractional-Order Chaotic Systems. When the parameters $\theta$ in system (3) are uncertain, the controlled response system and a parameter update law are expressed by

$$
\begin{gathered}
D^{q_{r}} Y=g(Y, \tilde{\theta})+u(X, Y, \theta), \\
D^{q_{r}} \tilde{\theta}=p(X, Y),
\end{gathered}
$$

where $\tilde{\theta}$ are unknown parameters to be estimated and $u(X, Y, \theta)$ are adaptive controller to be designed.

Definition 1 (MFPS). For the drive system (4) or system (6) and controlled system (7), it is said to be modified 
function projective synchronization (MFPS) if there exist controller $u(X, Y, \theta)$, parameter update law $p(X, Y)$, and a scaling function matrix $\Lambda(t)$, such that

$$
\begin{gathered}
\lim _{t \rightarrow+\infty}\|e\|=\lim _{t \rightarrow+\infty}\|Y-\Lambda(t) X\|=0, \\
\lim _{t \rightarrow+\infty}\left\|e_{\theta}\right\|=\lim _{t \rightarrow+\infty}\|\widetilde{\theta}-\theta\|=0,
\end{gathered}
$$

where $\|$. $\|$ is the Euclidean norm, $\Lambda(t)=$ $\operatorname{diag}\left(\alpha_{1}(t), \alpha_{2}(t), \ldots, \alpha_{n}(t)\right)$ and $\alpha_{i}(t)(i=1,2, \ldots, n)$ are continuous bounded functions. $e=\left(e_{1}, \ldots, e_{n}\right)^{T},\left(e_{i}=\right.$ $\left.Y_{i}-\alpha_{i}(t) X_{i}(i=1,2, \ldots, n)\right)$, and $e_{\theta}=\left(e_{\theta 1}, \ldots, e_{\theta k}\right)^{T}\left(e_{\theta i}=\right.$ $\left.\tilde{\theta}_{i}-\theta_{i}(i=1,2, \ldots, k)\right)$ are called the MFPS error vector.

Remark 2. It is easy to see that function projective synchronization and modified projective synchronization are the special cases of modified function projective synchronization where $\alpha_{1}(t)=\alpha_{2}(t)=\cdots=\alpha_{n}(t)=\alpha(t)$ and $\alpha_{1}(t)=$ $\alpha_{1}, \alpha_{2}(t)=\alpha_{2}, \ldots, \alpha_{n}(t)=\alpha_{n}$, respectively.

Next, we will discuss how to choose the controller and the parameter update laws. Let the controller $u(X, Y, \theta)=$ $u_{1}(X, \theta)+u_{2}(X, Y, \theta)$ and we choose

$$
u_{1}(X, \theta)=D^{q_{r}}(\Lambda(t) X)-g(\Lambda(t) X, \theta)
$$

where $\theta$ is the true values of the "unknown" parameters. For the fractional-order part $D^{q_{r}}(\Lambda(t) X)$ contained in controller, we can refer to the properties of fractional-order derivative; that is, the derivative is linear operation. Moreover, $\Lambda(t)$ is a function about $t$ and can be regarded as constant for derivative $D_{X}^{q_{r}}$. Therefore, we can obtain $D^{q_{r}}(\Lambda(t) X)=$ $\Lambda(t) D^{q_{r}} X$, which can be simulated the same as fractionalorder system.

Then, response system (7) can be rewritten as

$$
\begin{aligned}
D^{q_{r}} Y= & g(Y, \widetilde{\theta})+D^{q_{r}}(\Lambda(t) X) \\
& -g(\Lambda(t) X, \theta)+u_{2}(X, Y, \theta) .
\end{aligned}
$$

According to Definition 1 , we have the error vector as $e=$ $Y-\Lambda(t) X$, so we can obtain

$$
\begin{aligned}
D^{q_{r}} e= & g(Y, \widetilde{\theta})-g(\Lambda(t) X, \theta) \\
& +u_{2}(X, Y, \theta)
\end{aligned}
$$

Here, we assume that there exists a function $b_{1}(X, Y, \theta)$ such that

$$
g(Y, \widetilde{\theta})-g(\Lambda(t) X, \theta)=b_{1}(X, Y, \theta)\left(\begin{array}{c}
e \\
e_{\theta}
\end{array}\right),
$$

where $b_{1}(X, Y, \theta)$ is an $n \times(n+k)$ real matrix and $\left(\begin{array}{c}e \\ e_{\theta}\end{array}\right)=$ $\left(e_{1}, \ldots, e_{n}, e_{\theta 1}, \ldots, e_{\theta k}\right)$ is a real matrix. Many fractional-order chaotic (hyperchaotic) systems satisfy this assumption [14$18]$.
Now, vector $u_{2}(X, Y, \theta)$ and parameter update law are chosen as

$$
\begin{gathered}
u_{2}(X, Y, \theta)=b_{2}(X, Y, \theta)\left(\begin{array}{c}
e \\
e_{\theta}
\end{array}\right), \\
D^{q_{r}} \tilde{\theta}=D^{q_{r}} e_{\theta}=b_{3}(X, Y)\left(\begin{array}{c}
e \\
e_{\theta}
\end{array}\right),
\end{gathered}
$$

where $b_{2}(X, Y, \theta)$ is an $n \times(n+k)$ real matrix to be designed and $b_{3}(X, Y, \theta)$ is a $k \times(n+k)$ real matrix to be designed.

From (12)-(15), we can yield

$$
\begin{aligned}
\left(\begin{array}{c}
D^{q_{r}} e \\
D^{q_{r}} e_{\theta}
\end{array}\right) & =\left(\begin{array}{c}
b_{1}(X, Y, \theta)+b_{2}(X, Y, \theta) \\
b_{3}(X, Y)
\end{array}\right)\left(\begin{array}{c}
e \\
e_{\theta}
\end{array}\right) \\
& =b(X, Y, \theta)\left(\begin{array}{c}
e \\
e_{\theta}
\end{array}\right) .
\end{aligned}
$$

Now the MFPS problem between drive system (4) or system (6) and controlled system (7) has been transformed into the following problem: choose suitable real matrix $b_{2}(X, Y, \theta)$ and $b_{3}(X, Y)$ such that system (16) is asymptotically stable to zero.

Theorem 3. If the elements $b_{i j}(i, j=1,2, \ldots, n+k)$ of $(n+$ $k) \times(n+k)$ matrix $b(X, Y, \theta)$ satisfy the following conditions:

$$
\begin{aligned}
& \text { (1) } b_{i j}=-b_{j i} \quad(i \neq j) \\
& \text { (2) } b_{i i} \leq 0 \text { (not all } b_{i i} \text { are equal to zero), }
\end{aligned}
$$

then the MFPS between system (4) or system (6) and system (7) can be achieved.

Proof. Suppose that $\lambda$ is one of the eigenvalues of matrix $b(X, Y, \theta)$ and the corresponding nonzero eigenvector is $\beta$; that is,

$$
b(X, Y, \theta) \beta=\lambda \beta \text {. }
$$

Taking conjugate transpose on both sides of the above equation, one can yield

$$
\overline{[b(X, Y, \theta) \beta]^{T}}=\bar{\lambda} \beta^{H} .
$$

From (17) multiplied left by $\beta^{H}$ plus (18) multiplied right by $\beta$, we derive that

$$
\beta^{H}\left(b(X, Y, \theta)+b(X, Y, \theta)^{H}\right) \beta=\beta^{H}(\lambda+\bar{\lambda}) \beta .
$$

So, $\lambda+\bar{\lambda}=\beta^{H}\left(b(X, Y, \theta)+b(X, Y, \theta)^{H}\right) \beta / \beta^{H} \beta$. Because $b_{i j}=-b_{j i}(i \neq j)$ for matrix $b(X, Y, \theta)$, it is easy to get that

$$
\lambda+\bar{\lambda}=\beta^{H}\left(\begin{array}{cccc}
2 b_{11} & 0 & \cdots & 0 \\
0 & 2 b_{22} & \cdots & 0 \\
0 & 0 & \cdots & 0 \\
0 & 0 & 0 & 2 b_{n n}
\end{array}\right) \beta \times\left(\beta^{H} \beta\right)^{-1} .
$$

Since $b_{i i} \leq 0$ and all $b_{i i}$ are not equal to zero, we can obtain

$$
\lambda+\bar{\lambda} \leq 0
$$


From (21), we have $|\arg \lambda[b(X, Y, \theta)]| \geq \pi / 2>q_{r} \pi / 2$.

According to the stability theory of fractional-order systems [30], we can yield that system (16) is asymptotically stable. That is, $\lim _{t \rightarrow+\infty}\|e\|=\lim _{t \rightarrow+\infty}\left\|e_{\theta}\right\|=0$.

The proof is completed.

Therefore, for the drive system (4) or (6) and controlled system (7), the MFPS can be achieved and the uncertain parameters will be estimated.

Remark 4. Theorem 3 will still apply to the condition that the parameters of response system are known. In this case, $b_{1}(X, Y, \theta)$ and $b_{2}(X, Y, \theta)$ are all $n \times n$ real matrix and $b(X, Y, \theta)=b_{1}(X, Y, \theta)+b_{2}(X, Y, \theta)$. The corresponding examples will be illustrated in Section 3.2.

Remark 5. The main difference in the design of the controller between this paper and [28] is that the controller in [28] is based on decomposing the drive and response system into linear parts and nonlinear parts, which does not apply to all fractional-order systems. The controller in this paper is general for all fractional-order systems.

\section{Illustrative Examples}

In this section, to demonstrate the effectiveness of the proposed MFPS scheme for different dimension fractional-order chaotic systems, two numerical examples are respectively used to discuss two kinds of cases: the reduced order synchronization with $m>n$ and the increased order synchronization with $m<n$. These examples include hyperchaotic system drives chaotic system and 3-dimension chaotic system drives 4-dimension chaotic system. And a predictorcorrector scheme [31] is chosen for the following simulations of the fractional-order differential equations.

3.1. Reduced-Order MFPS with $m>n$. In this subsection, fractional-order hyperchaotic Lorenz system [17] as drive and fractional-order Chen system [16] as response are chosen to obtain the reduced-order MFPS behavior of fractional-order chaotic systems with unknown parameters.

The fractional-order hyperchaotic Lorenz dynamical differential equation can be given by

$$
\begin{gathered}
D^{q} x_{1}=\sigma\left(x_{2}-x_{1}\right)+x_{4}, \\
D^{q} x_{2}=\alpha x_{1}-x_{2}-x_{1} x_{3}, \\
D^{q} x_{3}=x_{1} x_{2}-\beta x_{3}, \\
D^{q} x_{3}=-x_{2} x_{3}+\gamma x_{4},
\end{gathered}
$$

where system parameters are chosen as $(\sigma, \alpha, \beta, \gamma)=$ $(10,28,8 / 3,-1)$. The fractional-order system exhibits hyperchaotic behavior for $q=0.98$.

In order to investigate the reduced-order MFPS behavior between hyperchaotic Lorenz system and Chen system, we assume that the $x_{1}-x_{2}-x_{3}$ projection of system (22) is the drive system and it can be presented in the form of

$$
\begin{gathered}
D^{q} x_{1}=\sigma\left(x_{2}-x_{1}\right)+x_{4}, \\
D^{q} x_{2}=\alpha x_{1}-x_{2}-x_{1} x_{3}, \\
D^{q} x_{3}=x_{1} x_{2}-\beta x_{3} .
\end{gathered}
$$

The controlled fractional-order Chen system with uncertain parameters is defined as

$$
\left(\begin{array}{l}
D^{q} y_{1} \\
D^{q} y_{2} \\
D^{q} y_{3}
\end{array}\right)=\left(\begin{array}{c}
\tilde{a}\left(y_{2}-y_{1}\right) \\
(\widetilde{c}-\widetilde{a}) y_{1}+\widetilde{c} y_{2}-y_{1} y_{3} \\
y_{1} y_{2}-\widetilde{b} y_{3}
\end{array}\right)+u(X, Y, \theta),
$$

where $\theta=(a, b, c)=(35,3,27)$. When $q \geq 0.83$, the fractional-order Chen system shows chaotic characteristic.

According to the above discussion, we can obtain

$b_{1}(X, Y, \theta)$

$$
=\left(\begin{array}{cccccc}
-a & a & 0 & y_{2}-y_{1} & 0 & 0 \\
c-a-\alpha_{3}(t) x_{3} & c & -y_{1} & -y_{1} & 0 & y_{1}+y_{2} \\
\alpha_{2}(t) x_{2} & y_{1} & -b & 0 & -y_{3} & 0
\end{array}\right) .
$$

Therefore, the possible parameter update law and real matrix $b_{2}(X, Y, \theta)$ are chosen as

$$
\begin{gathered}
D^{q} \widetilde{a}=\left(y_{1}-y_{2}\right) e_{1}+y_{1} e_{2}, \\
D^{q} \widetilde{b}=y_{3} e_{3}, \\
D^{q} \widetilde{c}=-\left(y_{1}+y_{2}\right) e_{2}, \\
b_{2}(X, Y, \theta)=\left(\begin{array}{cccccc}
0 & 0 & 0 & 0 & 0 & 0 \\
\alpha_{3}(t) x_{3}-c & -c-1 & 0 & 0 & 0 & 0 \\
-\alpha_{2}(t) x_{2} & 0 & 0 & 0 & 0 & 0
\end{array}\right) .
\end{gathered}
$$

Consequently, it is easy to obtain

$b(X, Y, \theta)$

$$
=\left(\begin{array}{cccccc}
-a & a & 0 & y_{2}-y_{1} & 0 & 0 \\
-a & -1 & -y_{1} & -y_{1} & 0 & y_{1}+y_{2} \\
0 & y_{1} & -b & 0 & -y_{3} & 0 \\
y_{1}-y_{2} & y_{1} & 0 & 0 & 0 & 0 \\
0 & 0 & y_{3} & 0 & 0 & 0 \\
0 & -\left(y_{1}+y_{2}\right) & 0 & 0 & 0 & 0
\end{array}\right) .
$$

Obviously, the elements in $b(X, Y, \theta)$ satisfy conditions (2)-(3) given in Theorem 3. Thus, the MFPS between projected system (23) and response system (24) can be achieved and the uncertain parameters can be exactly estimated. Here, choose scale function vector $\Lambda(t)=(1+\sin (t), 2+t, 2+0.8 \cos (t))$. The initial conditions are $\left(x_{1}(0), x_{2}(0), x_{3}(0), x_{4}(0)\right)=(2,-1,1,1)$, $\left(y_{1}(0), y_{2}(0), y_{3}(0)\right)=(1,-2,3)$ and initial values of unknown parameters are $(\widetilde{a}(0), \widetilde{b}(0), \widetilde{c}(0))=(30,1,25)$. 
The corresponding numerical results are shown in Figures 1-2. Figure 1 displays the error state trajectories of the drive system (23) and response system (24). Figure 2 shows that the estimated values of parameters in system (24) converge to their true values $a=35, b=3$, and $c=27$ as $t \rightarrow \infty$.

These results show that the MFPS between hyperchaotic system (22) and chaotic system (24) and parameters identification have been achieved with the adaptive control law $u(X, Y, \theta)$ and the parameter update laws (26).

3.2. Added-Order MFPS with $m<n$. It is assumed that the fractional-order chaotic Arneodo system [15] drives the fractional-order hyperchaotic Lü system [18] with known parameters. The order of Arneodo system is increased to synchronize to 4-dimensional Lü system.

The fractional-order Arneodo system is defined as

$$
\begin{gathered}
D^{q} x_{1}=x_{2}, \\
D^{q} x_{2}=x_{3}, \\
D^{q} x_{3}=\alpha x_{1}+\beta x_{2}+\gamma x_{3}+\delta x_{1}^{3} .
\end{gathered}
$$

When $(\alpha, \beta, \gamma, \delta)=(5.5,-3.5,-1,-1)$ and $q=0.95$, the Arneodo system exhibits chaotic behavior.

The equations of the 4-dimensional fractional-order Lü system are

$$
\begin{gathered}
D^{q} y_{1}=a\left(y_{2}-y_{1}\right)+y_{4}, \\
D^{q} y_{2}=c y_{2}-y_{1} y_{3}, \\
D^{q} y_{3}=y_{1} y_{2}-b y_{3}, \\
D^{q} y_{4}=y_{1} y_{3}-y_{4} .
\end{gathered}
$$

It has been shown that system (30) will exhibit hyperchaotic behavior when $q=0.95$ and $a=36, b=3$, and $c=20$.

Based on the above method, we construct an auxiliary state variable and its fractional derivative $D^{q} x_{4}=x_{1}\left(1-x_{2}\right)$ for Arneodo system; the drive system; after adding order can be written as

$$
\begin{gathered}
D^{q} x_{1}=x_{2}, \\
D^{q} x_{2}=x_{3}, \\
D^{q} x_{3}=\alpha x_{1}+\beta x_{2}+\gamma x_{3}+\delta x_{1}^{3}, \\
D^{q} x_{4}=x_{1}\left(1-x_{2}\right) .
\end{gathered}
$$
that

According to the discussion in Section 2, we can obtain

$$
b_{1}(X, Y, \theta)=\left(\begin{array}{cccc}
-a & a & 0 & 1 \\
-\alpha_{3}(t) x_{3} & c & -y_{1} & 0 \\
\alpha_{2}(t) x_{2} & y_{1} & -b & 0 \\
\alpha_{3}(t) x_{3} & 0 & y_{1} & -1
\end{array}\right) \text {. }
$$
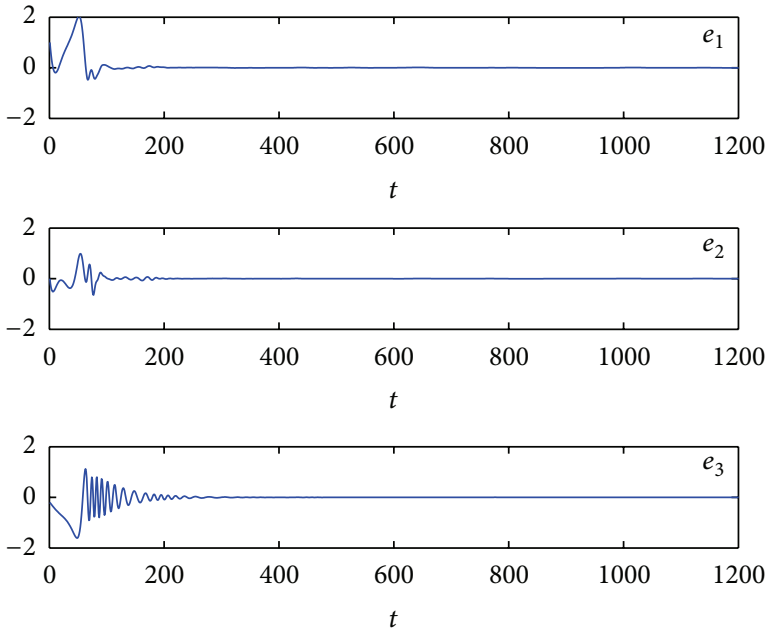

FIGURE 1: The time evolutions of MFPS errors between system (23) and system (24).

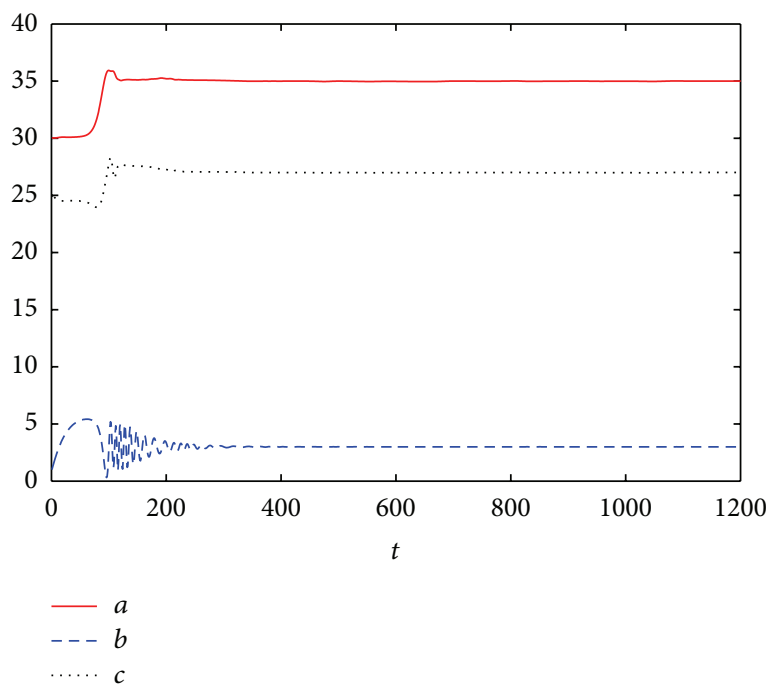

Figure 2: Changing parameters $a, b$, and $c$ of Chen system (24) with time $t$.

Now, we can choose

$$
b_{2}(X, Y, \theta)=\left(\begin{array}{cccc}
0 & 0 & 0 & 0 \\
-a+\alpha_{3}(t) x_{3} & -1-c & 0 & 0 \\
-\alpha_{2}(t) x_{2} & 0 & 0 & 0 \\
-1-\alpha_{3}(t) x_{3} & 0 & -y_{1} & 0
\end{array}\right) .
$$

So,

$$
b(X, Y, \theta)=\left(\begin{array}{cccc}
-a & a & 0 & 1 \\
-a & -1 & -y_{1} & 0 \\
0 & y_{1} & -b & 0 \\
-1 & 0 & 0 & -1
\end{array}\right)
$$

According to the above theorem, the MFPS between 3dimensional Arneodo system and 4-dimensional Lü system can be achieved via added order. We have performed some numerical simulations to verify the above theoretical analysis. 

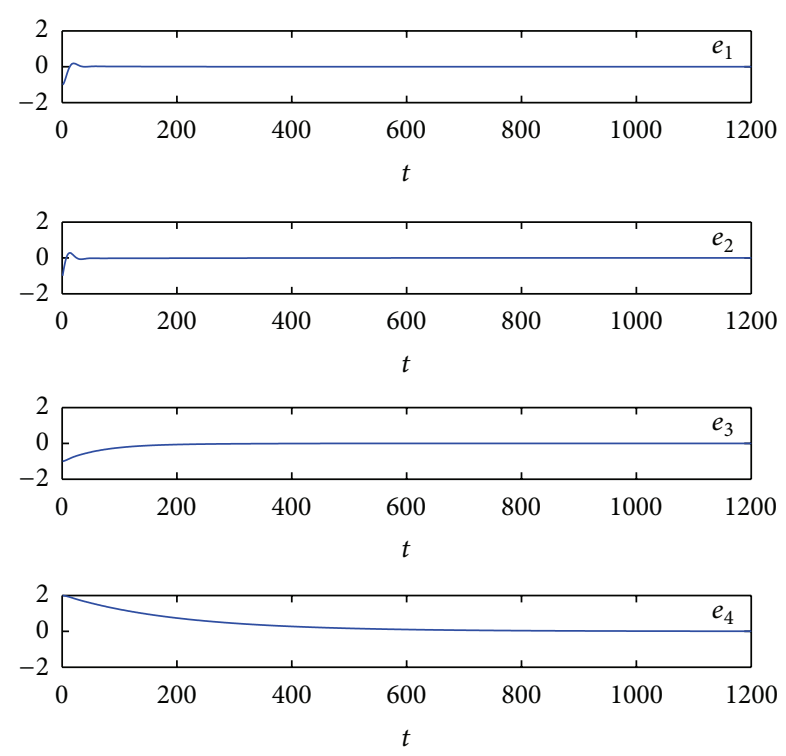

FIgURE 3: The MFPS errors between added-order system (31) and system (30).

For example, choose $\Lambda(t)=(1+\sin (t), 2 t+3,2+$ $0.8 \cos (t), 4 \cos (t))$. The corresponding numerical results are shown in Figure 3.

In Figure 3, the initial conditions for drive system (30) and response system $(29)$ are $\left(x_{1}(0), x_{2}(0), x_{3}(0), x_{4}(0)\right)=$ $(1,2,1,2)$ and $\left(y_{1}(0), y_{2}(0), y_{3}(0), y_{4}(0)\right)=(2,7,3,6)$, respectively. From the results in Figure 3 , we can see that addedorder 3-dimensional system and 4-dimensional system can synchronize up to a scale function matrix.

\section{Conclusions}

In summary, the adaptive reduced-order or added-order MFPS of chaotic systems with different dimensions is discussed. When the order of drive system is slightly large, the drive system is order-reduced to synchronize response. If drive system has lower dimension, some auxiliary states are added to increase the dimension of drive system. According to the stability theorems of linear fractional-order systems, an adaptive controller and a parameter update law are presented to synchronize different dimensional systems. It is shown that the response system can not only synchronize with the projection of the drive system but also synchronize with the drive system that includes auxiliary state. This technique has been successfully applied to two examples: fractional-order hyperchaotic Lorenz system drives fractional-order Chen system with unknown parameters; fractional-order Arneodo system drives the fractional-order hyperchaotic Lü system with known parameters.

In this paper, we propose a general method to achieve MFPS of fractional-order chaotic systems even though their dimensions are distinct. The method can be extended to the condition that the derivative orders of drive and response systems are unequal or the fractional-order system with incommensurate orders.

\section{Acknowledgments}

This research was supported by the National Natural Science Foundation of China (Grant nos. 61374178 and 61202085), the Liaoning Provincial Natural Science Foundation of China (Grant no. 201202076), the Specialized Research Fund for the Doctoral Program of Higher Education (Grant no. 20120042120010), and the Ph.D. Startup Foundation of Liaoning Province, China (Grant nos. 20111001, 20121001, and 20121002).

\section{References}

[1] B. Blasius, A. Huppert, and L. Stone, "Complex dynamics and phase synchronization in spatially extended ecological system," Nature, vol. 399, pp. 354-359, 1999.

[2] M. Lakshmanan and K. Murali, Chaos in Nonlinear Oscillators: Controlling and Synchronization, World Scientific, Singapore, 1996.

[3] S. K. Han, C. Kerrer, and Y. Kuramoto, "Dephasing and bursting in coupled neural oscillators," Physical Review Letters, vol. 75, pp. 3190-3193, 1995.

[4] K. M. Cuomo and A. V. Oppenheim, "Circuit implementation of synchronized chaos with application to communication," Physical Review Letters, vol. 71, pp. 65-68, 1993.

[5] L. M. Pecora and T. L. Carroll, "Synchronization in chaotic systems," Physical Review Letters, vol. 64, no. 8, pp. 821-824, 1990.

[6] C. W. Wu and L. O. Chua, "A unified framework for synchronization and control of dynamical systems," International Journal of Bifurcation and Chaos in Applied Sciences and Engineering, vol. 4, no. 4, pp. 979-998, 1994.

[7] M. G. Rosenblum, A. S. Pikovsky, and J. Kurths, "Phase synchronization of chaotic oscillators," Physics Review Letters, vol. 76, pp. 1804-1807, 1996.

[8] S. Taherionl and Y. C. Lai, "Observability of lag synchronization of coupled chaotic oscillators," Physical Review E, vol. 59, pp. 6247-6250, 1999.

[9] Z. Y. Yan, "Q-S (lag or anticipated) synchronization backstepping scheme in a class of continuous-time hyperchaotic systems-a symbolic-numeric computation approach," Chaos, vol. 15, no. 2, Article ID 023902, 9 pages, 2005.

[10] R. Mainieri and J. Rehacek, "Projective synchronization in three-dimensional chaotic systems," Physics Review Letters, vol. 82, pp. 3042-3045, 1999.

[11] K. S. Sudheer and M. Sabir, "Adaptive modified function projective synchronization between hyperchaotic Lorenz system and hyperchaotic Lu system with uncertain parameters," Physics Letters A, vol. 373, no. 41, pp. 3743-3748, 2009.

[12] K. S. Sudheer and M. Sabir, "Switched modified function projective synchronization of hyperchaotic Qi system with uncertain parameters," Communications in Nonlinear Science and Numerical Simulation, vol. 15, pp. 4058-4064, 2010.

[13] J. R. Chen, L. C. Jiao, J. S. Wu et al., "Projective synchronization with different scale factors in a driven-response complex network and its application in image encryption," Nonlinear Analysis: Real World Applications, vol. 11, pp. 3045-3058, 2010.

[14] T. T. Hartley, C. F. Lorenzo, and H. K. Qammer, "Chaos in a fractional order Chua's system," IEEE Transactions on Circuits and Systems I, vol. 42, pp. 485-490, 1995. 
[15] J. G. Lu, "Chaotic dynamics and synchronization of fractionalorder Arneodo's systems," Chaos Solitons \& Fractals, vol. 26, pp. 1125-1133, 2005.

[16] C. Li and G. Peng, "Chaos in Chen's system with a fractional order," Chaos, Solitons \& Fractals, vol. 22, no. 2, pp. 443-450, 2004.

[17] X. Y. Wang and J. M. Song, "Synchronization of the fractional order hyperchaos Lorenz systems with activation feedback control," Communications in Nonlinear Science and Numerical Simulation, vol. 14, pp. 3351-3357, 2009.

[18] F. H. Min, Y. Yu, and C. J. Ge, "Circuit implementation and tracking control of the fractional-order hyper-chaotic Lü system," Acta Physica Sinica, vol. 58, no. 3, pp. 1456-1461, 2009.

[19] M. S. Tavazoei, M. Haeri, and N. Nazari., "Analysis of undamped oscillations generated by marginally stable fractional order systems," Signal Processing, vol. 88, pp. 2971-2978, 2008.

[20] S. K. Agrawal, M. Srivastava, and S. Das, "Synchronization of fractional order chaotic systems using active control method," Chaos, Solitons \& Fractals, vol. 45, pp. 737-752, 2012.

[21] R. X. Zhang and S. P. Yang, "Adaptive synchronization of fractional-order chaotic systems via a single driving variable," Nonlinear Dynamics, vol. 66, no. 4, pp. 831-837, 2011.

[22] J. G. Lu, "Nonlinear observer design to synchronize fractionalorder chaotic system via a scaler transmitted signal," Physica A, vol. 359, pp. 107-118, 2006.

[23] D.-Y. Chen, Y.-X. Liu, X.-Y. Ma, and R.-F. Zhang, "Control of a class of fractional-order chaotic systems via sliding mode," Nonlinear Dynamics, vol. 67, no. 1, pp. 893-901, 2012.

[24] P. Zhou and R. Ding, "Chaotic synchronization between different fractional-order chaotic systems," Journal of the Franklin Institute, vol. 348, no. 10, pp. 2839-2848, 2011.

[25] S. Y. Li and Z. M. Ge, "Generalized synchronization of chaotic systems with different orders by fuzzy logic constant controller," Expert Systems with Applications, vol. 38, pp. 2302-2310, 2011.

[26] R. Femat and J. Alvarez-Ramírez, "Synchronization of a class of strictly different chaotic oscillators," Physics Letters A, vol. 236, no. 4, pp. 307-313, 1997.

[27] D. Terman, N. Kopell, and A. Bose, "Dynamics of two mutually coupled slow inhibitory neurons," Physica D, vol. 117, no. 1-4, pp. 241-275, 1998.

[28] S. Wang, Y. G. Yu, and M. Diao, "Hybrid projective synchronization of chaotic fractional order systems with different dimensions," Physica A, vol. 389, pp. 4981-4988, 2010.

[29] S. Wang and Y. G. Yu, "Generalized projective synchronization of fractional order chaotic systems with different dimensions," Chinese Physics Letters, vol. 29, no. 2, Article ID 020505, 2012.

[30] D. Matignon, "Stability results of fractional differential equation with applications to control processing," in Computational Engineering in Systems Applications, pp. 963-968, 1996.

[31] K. Diethelm, N. J. Ford, and A. D. Freed, "A predictor-corrector approach for the numerical solution of fractional differential equations," Nonlinear Dynamics, vol. 29, no. 1-4, pp. 3-22, 2002. 


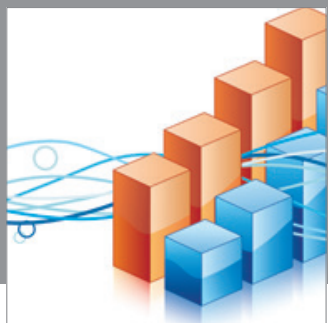

Advances in

Operations Research

mansans

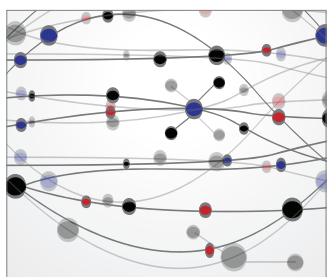

The Scientific World Journal
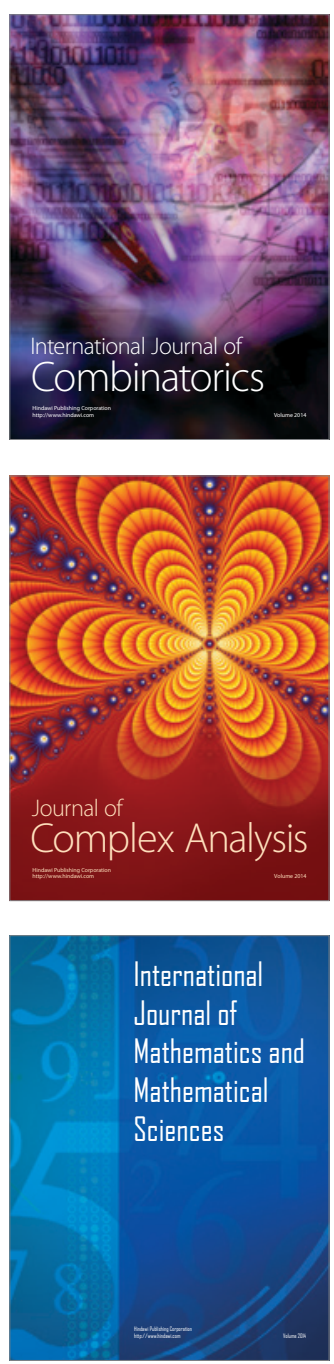
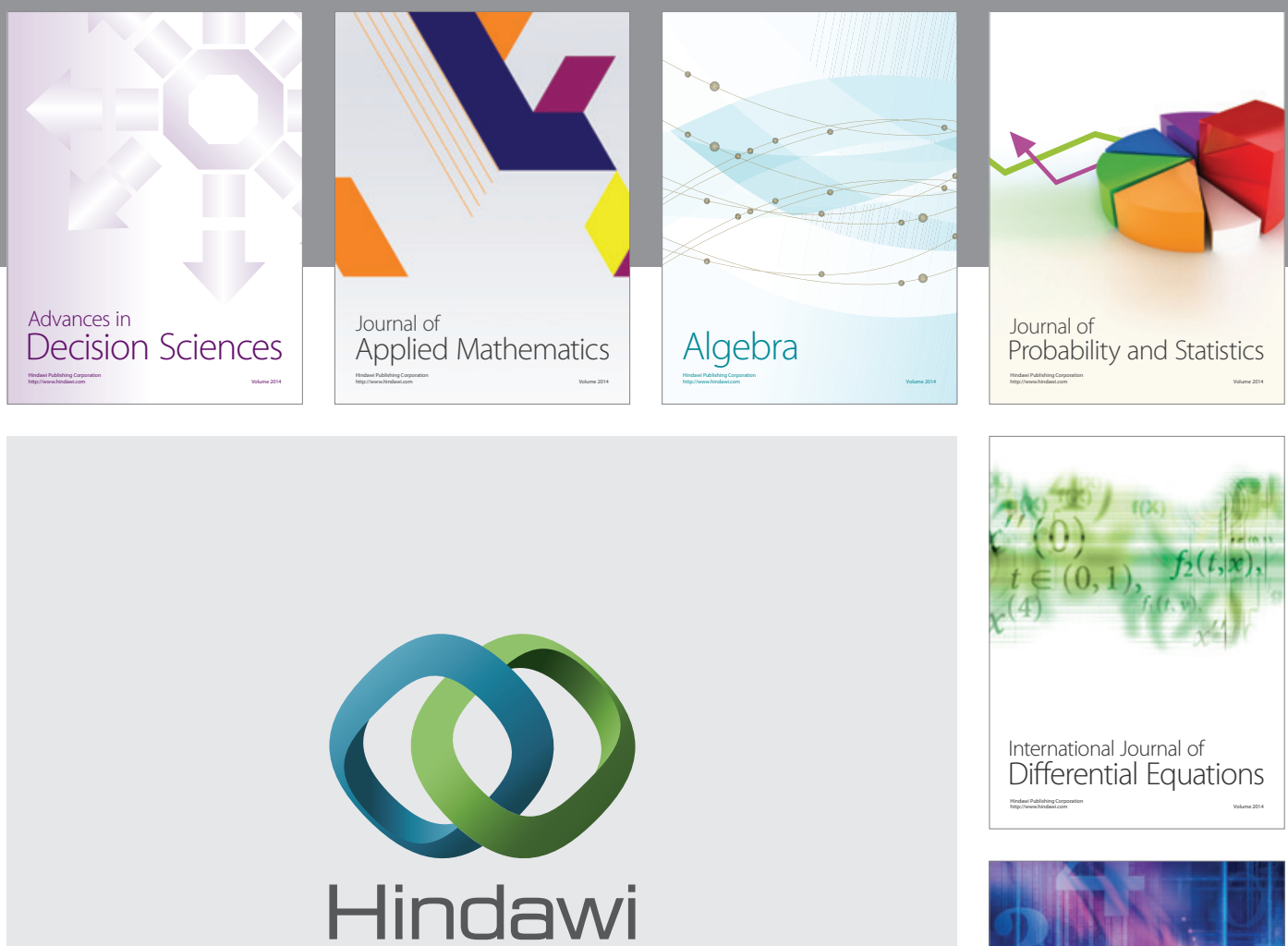

Submit your manuscripts at http://www.hindawi.com
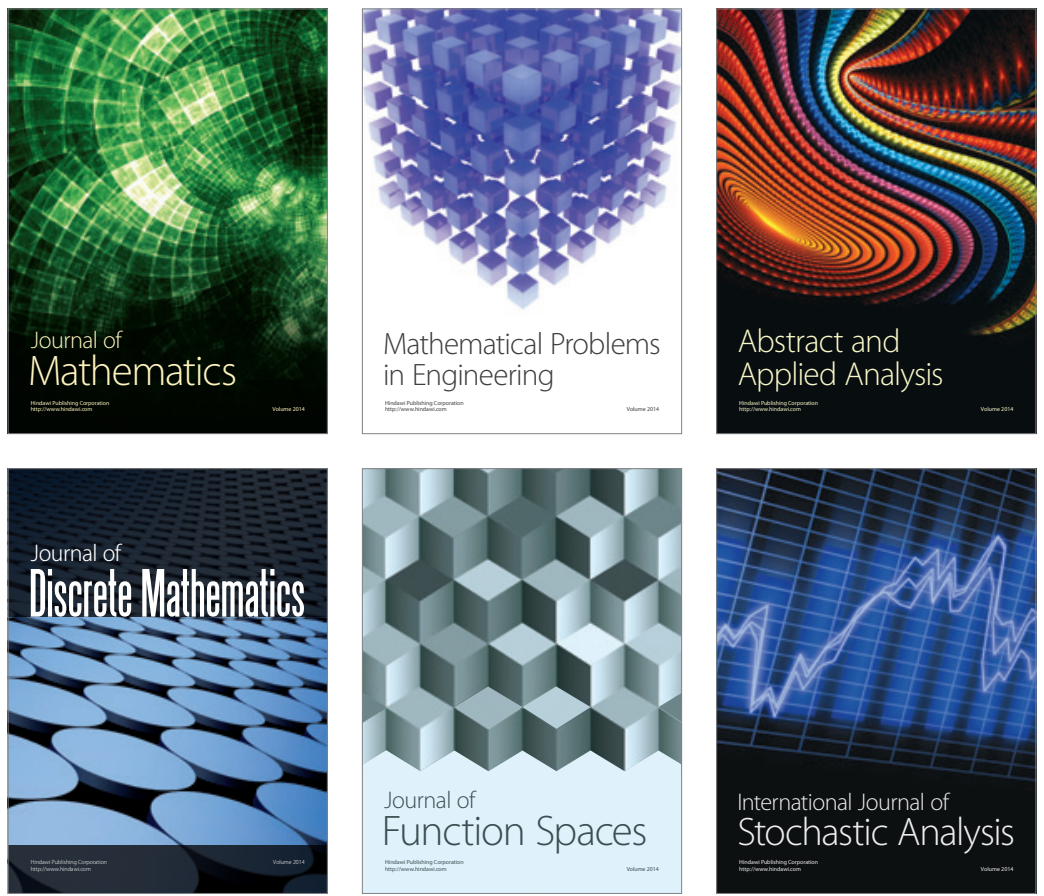

Journal of

Function Spaces

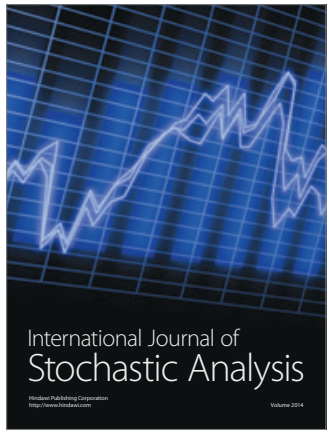

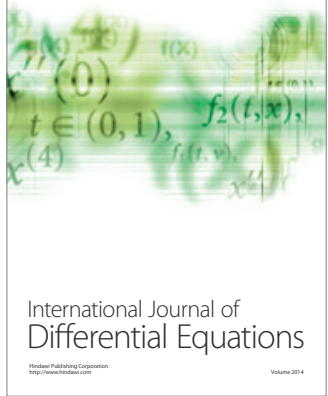
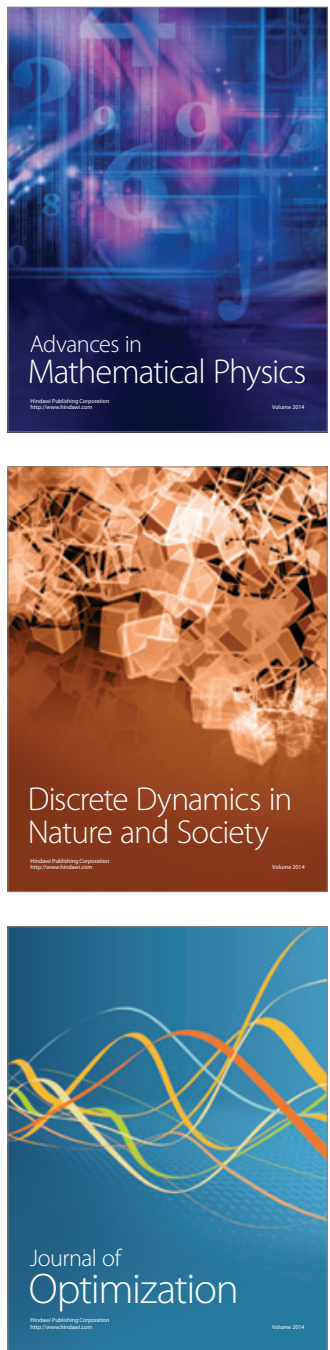